\title{
Combinatorial Random Walks on 3-Manifolds
}

\author{
Markus Banagl
}

\section{CONTENTS}

1. Introduction

2. The Mean Commute Time

3. Random Walks on Graphs

4. Triangulations of 3-Manifolds

5. Passage to Quartic Graphs

6. Electrical Resistance

7. The Algorithm

8. Experimental Results

Acknowledgments

References

2000 AMS Subject Classification: Primary 60B99;

Secondary 60G50, 57N10

Keywords: Statistical topology, 3-manifolds, random walks, electrical resistance
We define a combinatorial, discrete-time random walk on a closed, triangulated 3-manifold. As one varies the triangulation, keeping the number of tetrahedra fixed, the maximal mean commute time of the random walk becomes a random variable on a finite, uniform probability space of triangulations. Using computer experiments, we obtain empirical density functions for these random variables. The densities are then applied in developing Bayes-type heuristics that allow a walking entity, moving randomly in an unknown 3-manifold, to obtain probabilistic information about which manifold it might be moving in. Mean commute times are calculated via the effective electrical resistance of certain quartic graphs associated with the random walk. As a by-product, we define a topological invariant, the electrical resistance, of a 3-manifold, which we interpret as a refined complexity measure with values in the rational numbers.

\section{INTRODUCTION}

We investigate the statistical topology of 3-manifolds by posing the following question: To what extent is the topology of a manifold remembered by purely statistical properties of certain stochastic processes executed on the manifold? In the present paper, we shall focus on compact three-dimensional manifolds without boundary, and the stochastic process we will consider is a combinatorial, discrete-time random walk on the manifold. Our model for the random walk is based on the well-known fact due to Moise [Moise 52] that every compact 3-manifold $M$ can be triangulated by a simplicial complex $T$ with finitely many tetrahedra (see also [Bing 59]). Choose and fix such a $T$. From a tetrahedron, the walk proceeds across one of the tetrahedron's four 2-faces to the adjacent tetrahedron. The face is chosen uniformly at random. Note that since $M$ is a closed manifold, every two-simplex is indeed the face of precisely two tetrahedra.

We focus here on the mean commute time associated with such random walks, i.e., the expected number of

(c) A K Peters, Ltd. 1058-6458/2006 $\$ 0.50$ per page Experimental Mathematics 15:3, page 367 
steps that it takes to go from a tetrahedron $a$ to a tetrahedron $b$ and back to $a$. An important point to be emphasized is that the walking entity itself can, by maintaining a "flight log," calculate approximations to the mean commute times. The theorems and heuristics presented below can then be used by the walking entity to obtain information about which manifold it might be walking in.

Fix the manifold $M$ and the number $n$ of tetrahedra. Then there are finitely many triangulations of $M$ having $n$ tetrahedra (see Lemma 4.3). Associating to every such triangulation the maximal mean commute time of the random walk on it defines a random variable on the finite, uniform probability space of triangulations of $M$ with $n$ tetrahedra. Our main results are empirical density functions of these random variables, for certain manifolds and certain interesting values of $n$. The densities are calculated by executing computer experiments. ${ }^{1}$ Our algorithm generates a large number of triangulations of a fixed manifold at random and then calculates mean commute times for random walks on each of these triangulations by computing the effective electrical resistance of an associated quartic graph, using classical results from graph theory.

As a by-product of these investigations we define a topological invariant, the electrical resistance of a 3manifold, which we interpret as a refined complexity measure of the manifold. For example, $S^{3}$ has resistance 0.4 , and the 3-dimensional Klein bottle has resistance $0.8056 .^{2}$

The theoretical densities are not known at present, since we do not have complete lists of triangulations of 3-manifolds. Even if we did, they would be extremely long: it is known, for instance, that already the 2-sphere $S^{2}$ has $28,615,703,421,545$ triangulations with up to 23 vertices.

What conclusions can we draw from the empirical densities? At first, one probably expects statistical quantities associated with such random walks to reflect very little of the full topological structure of the manifold. It turns out, however, that in certain special situations, the mean commute time carries enough information to allow one to determine the manifold completely. We prove, for example, the following theorem, which appears as Theorem 8.1 in Section 8.1.

\footnotetext{
${ }^{1}$ The $\mathrm{C}$ code of this software is available at http://www.mathi. uni-heidelberg.de/ banagl/statisticaltopology/er.c.

${ }^{2}$ Throughout this paper, all numerical equalities are to be understood, of course, as decimal approximations.
}

Theorem Suppose an unknown manifold $M$ is triangulated with 27 tetrahedra. If the maximal mean commute time associated with the combinatorial random walk on this triangulation is greater than or equal to 88 , then $M$ is the 3-sphere.

In general, we are interested in calculating, for a given number of tetrahedra $n$ and a given threshold time $t$, the a posteriori probabilities

$$
\mathbb{P}(M \mid C \leq t),
$$

that is, the probability that the manifold one is walking in is $M$ after having observed that the commute time $C$ is less than or equal to $t$. We wish to calculate this in terms of the a priori probabilities $\mathbb{P}(M)$ (the probability that a 3 -manifold triangulation with $n$ tetrahedra triangulates $M$; for more information on this probability measure see Sections 4 and 5). As an illustration, the empirical density functions enable us to generate Bayestype results such as the following, which is Heuristic 8.2 in Section 8.1.

Heuristic. Suppose an unknown manifold $M$ is triangulated with 27 tetrahedra. Assume we know that the maximal mean commute time associated with the combinatorial random walk on this triangulation is less than or equal to 102 . Then $M$ is either the 3 -sphere or $S^{2} \rtimes S^{1}$ with probabilities

$$
\begin{aligned}
\mathbb{P}\left(S^{3} \mid C \leq 102\right) & =\frac{0.009 \mathbb{P}\left(S^{3}\right)}{0.009 \mathbb{P}\left(S^{3}\right)+\mathbb{P}\left(S^{2} \rtimes S^{1}\right)}, \\
\mathbb{P}\left(S^{2} \rtimes S^{1} \mid C \leq 102\right) & =\frac{\mathbb{P}\left(S^{2} \rtimes S^{1}\right)}{0.009 \mathbb{P}\left(S^{3}\right)+\mathbb{P}\left(S^{2} \rtimes S^{1}\right)} .
\end{aligned}
$$

Here $S^{2} \rtimes S^{1}$ denotes the 3-dimensional Klein bottle, i.e., the space obtained from $S^{2} \times[0,1]$ by antipodal identification of $S^{2} \times\{0\}$ and $S^{2} \times\{1\}$. Thus we learn that knowing that the commute time is relatively small gives high weight to $S^{2} \rtimes S^{1}$. The following is Heuristic 8.4 in Section 8.2.

Heuristic. Suppose an unknown manifold $M$ is triangulated with 40 tetrahedra. Let $C$ denote the maximal mean commute time associated with the combinatorial random walk on this triangulation. Then:

1. $C<120$ or $C>135$.

2. If $C>135$, then $M$ is $S^{3}, S^{2} \rtimes S^{1}$, or $S^{2} \times S^{1}$. 
3. If $C<120$, then $M=\mathbb{R} P^{3}$.

4. Assume we know for instance that $C \leq 162$. Then $M$ is $S^{3}, S^{2} \rtimes S^{1}, S^{2} \times S^{1}$, or $\mathbb{R} P^{3}$ with probabilities

$$
\begin{aligned}
\mathbb{P}\left(S^{3} \mid C \leq 162\right) & =0.0023 \mathbb{P}\left(S^{3}\right) / p, \\
\mathbb{P}\left(S^{2} \rtimes S^{1} \mid C \leq 162\right) & =0.0957 \mathbb{P}\left(S^{2} \rtimes S^{1}\right) / p, \\
\mathbb{P}\left(S^{2} \times S^{1} \mid C \leq 162\right) & =0.0777 \mathbb{P}\left(S^{2} \rtimes S^{1}\right) / p, \\
\mathbb{P}\left(\mathbb{R} P^{3} \mid C \leq 162\right) & =\mathbb{P}\left(\mathbb{R} P^{3}\right) / p,
\end{aligned}
$$

where

$$
\begin{aligned}
p= & 0.0023 \mathbb{P}\left(S^{3}\right)+0.0957 \mathbb{P}\left(S^{2} \rtimes S^{1}\right) \\
& +0.0777 \mathbb{P}\left(S^{2} \times S^{1}\right)+\mathbb{P}\left(\mathbb{R} P^{3}\right) .
\end{aligned}
$$

From the previous heuristic we learn that knowing that the commute time is relatively small gives high weight to projective space. For instance, if we draw a sample out of all triangulations with 40 tetrahedra (containing possibly several copies of the same triangulation) so that the entropy of the partition $S^{3}, S^{2} \rtimes S^{1}, S^{2} \times S^{1}$, $\mathbb{R} P^{3}$ is maximized, then

$$
\begin{aligned}
\mathbb{P}\left(S^{3} \mid C \leq 162\right) & =0.00196, \\
\mathbb{P}\left(S^{2} \rtimes S^{1} \mid C \leq 162\right) & =0.08140, \\
\mathbb{P}\left(S^{2} \times S^{1} \mid C \leq 162\right) & =0.06609, \\
\mathbb{P}\left(\mathbb{R} P^{3} \mid C \leq 162\right) & =0.85056 .
\end{aligned}
$$

These heuristics are found using, in addition to the empirical densities, results on the combinatorics of triangulations of 3-manifolds and their $f$-vectors established in discrete geometry (especially the powerful results of Walkup [Walkup 70]).

\section{THE MEAN COMMUTE TIME}

Let $I$ be a finite state-space and $\left(X_{t}\right)_{t=0,1,2, \ldots}$ a discretetime Markov chain with underlying probability space $(\Omega, \mathcal{F}, \mathbb{P}), X_{t}: \Omega \rightarrow I$, and transition matrix $P=$ $\left(p_{i j}\right)_{i, j \in I}, p_{i j}=\mathbb{P}\left(X_{1}=j \mid X_{0}=i\right)$. Given $i \in I$, we write $\mathbb{P}_{i}(A)$ for the conditional probability $\mathbb{P}\left(A \mid X_{0}=i\right)$. The hitting time of $j \in I$ is the random variable

$$
H_{j}: \Omega \longrightarrow\{0,1,2, \ldots\} \cup\{\infty\}
$$

given by

$$
H_{j}(\omega)=\inf \left\{t \geq 0: X_{t}(\omega)=j\right\} .
$$

The mean hitting time for $j$ starting from $i \in I$, i.e., the mean time taken for $\left(X_{t}\right)_{t=0,1,2, \ldots}$ to reach $j$ provided $X_{0}=i$, is

$$
\mathbb{E}_{i}\left(H_{j}\right)=\sum_{k=1}^{\infty} \mathbb{P}_{i}\left(H_{j} \geq k\right) .
$$

The mean commute time between $i$ and $j$ is

$$
C(i, j)=\mathbb{E}_{i}\left(H_{j}\right)+\mathbb{E}_{j}\left(H_{i}\right) .
$$

This quantity expresses the expected number of steps that it takes the chain to go from $i$ to $j$ and back to $i$. The maximal mean commute time is defined to be

$$
C_{\max }=\max _{i, j} C(i, j) .
$$

\section{RANDOM WALKS ON GRAPHS}

A graph $G=(V, E)$ with finite set of nodes $V$ and finite set of edges $E$ is assumed to be undirected, without multiple edges and without self-loops. We write $n=|V|$ for the number of nodes and $m=|E|$ for the number of edges. In our terminology, we will distinguish between "vertex," which is understood to be a 0-simplex in a simplicial complex, and "node," which refers to an element of $V$. The graph $G$ is called $d$-regular if the degree of every node is $d$. Note that for such a graph, we have the relation

$$
m=\frac{1}{2} d n .
$$

The class of quartic, i.e., 4-regular, graphs will play a distinguished role in this paper, for we will describe in Section 5 a construction associating a quartic graph to any given triangulation of a closed 3-manifold.

Given a graph $G$, there is a natural definition of a Markov chain on $V=I$. A discrete-time random walk on $G$ is the Markov chain with transition matrix

$$
p_{v w}= \begin{cases}1 / d_{v} & \text { if }(v, w) \text { is an edge } \\ 0 & \text { if not }\end{cases}
$$

where $d_{v}$ is the degree of the node $v$. The corresponding stationary distribution is

$$
\pi_{v}=\frac{d_{v}}{2 m} .
$$

Thus, as described in the previous section, we may consider the mean commute time $C_{G}(v, w)$ between any two nodes $v, w$ and the maximal mean commute $C(G):=$ $C_{\max }$ of a graph $G$. 


\section{TRIANGULATIONS OF 3-MANIFOLDS}

Let $M^{3}$ be a three-dimensional manifold. In the present paper, we work exclusively with connected, closed $M$, i.e., 3-manifolds are compact and have no boundary. The literature on 3-manifolds uses mainly two different concepts of triangulation: one triangulates $M$ by a simplicial complex (in the sense of [Munkres 84, Section 1.3]), or one triangulates $M$, more generally, by a simplicial cell complex. The difference is that in a simplicial complex a simplex is uniquely determined by its vertices. This is not the case in a simplicial cell complex. For example, one needs five tetrahedra to triangulate the 3 -sphere $S^{3}$ by a simplicial complex (take the boundary of a standard 4-simplex), but only one tetrahedron is required to triangulate it as a simplicial cell complex. This paper uses only triangulations by simplicial complexes. The reason for this choice is explained in Section 5.

For a triangulation $T$, let $f_{d}, d=0,1,2,3$, denote the number of $d$-dimensional simplices in $T$. The quadruple $f=\left(f_{0}, f_{1}, f_{2}, f_{3}\right)$ is called the $f$-vector of $T$. By Poincaré duality, the Euler characteristic $\chi(M)$ of $M$ vanishes, $\chi(M)=f_{0}-f_{1}+f_{2}-f_{3}=0$. In a manifold triangulation, every 2 -simplex is a face of precisely two tetrahedra. (This is in fact still true if the space is only a pseudomanifold.) Hence $2 f_{2}=4 f_{3}$. Summarizing, an $f$-vector of a 3 -manifold triangulation has the form

$$
f=\left(f_{0}, f_{1}, 2\left(f_{1}-f_{0}\right), f_{1}-f_{0}\right) .
$$

Two theorems of Walkup [Walkup 70] will be used in establishing our heuristics:

Theorem 4.1. With the exception of $S^{3}, S^{2} \rtimes S^{1}$, and $S^{2} \times S^{1}$, which have minimal triangulations with 5, 9, and 10 vertices, respectively, every other triangulated 3 manifold has at least 11 vertices.

Theorem 4.2. For every 3-manifold $M$, there is an integer $\gamma(M)$ such that

$$
f_{1} \geq 4 f_{0}+\gamma(M)
$$

for every triangulation of $M$. One has

$$
\begin{aligned}
\gamma\left(S^{3}\right) & =-10, \\
\gamma\left(S^{2} \rtimes S^{1}\right) & =\gamma\left(S^{2} \times S^{1}\right)=0, \\
\gamma\left(\mathbb{R} P^{3}\right) & =7,
\end{aligned}
$$

with $\gamma(M) \geq 8$ for every other 3-manifold $M$.
Let $\operatorname{Triang}_{n}(M)$ be the set of isomorphism classes of abstract simplicial complexes $T$ with $n$ tetrahedra whose topological realization $|T|$ is homeomorphic to $M$.

Lemma 4.3. The set $\operatorname{Triang}_{n}(M)$ is finite.

Proof: This is implied by Theorem 4.2. The details are as follows: For any triangulation $T \in \operatorname{Triang}_{n}(M)$, let $f=\left(f_{0}, f_{1}, f_{2}, f_{3}=n\right)$ denote its $f$-vector. According to Walkup's theorem, $f_{1} \geq 4 f_{0}-10$ for any $T$. By equation $(4-1), f_{3}=f_{1}-f_{0}$. Consequently, the number of vertices is bounded above in terms of the number of tetrahedra:

$$
f_{0} \leq \frac{n+10}{3} \text {. }
$$

Let $V$ be the set of vertices in $T$. Then $T$ can be uniquely described by specifying its tetrahedra, that is, by specifying a set with $n$ elements, each of which is a set of four elements (the vertices of the tetrahedron) chosen from $V$. Therefore,

$$
\left|\operatorname{Triang}_{n}(M)\right| \leq\left(\begin{array}{c}
\left(f_{0}\right. \\
4 \\
n
\end{array}\right) \leq\left(\begin{array}{c}
\left(\frac{1}{3}(n+10)\right. \\
4 \\
n
\end{array}\right) .
$$

To relate two triangulations of a manifold $M^{3}$ to each other, we use Pachner's results [Pachner 78, Pachner 87]. Let $M^{d}$ be a $d$-dimensional manifold triangulated by a simplicial complex $T$. For $0 \leq i \leq d$, Pachner defines a bistellar $i$-move as follows: Choose any $(d-i)$-simplex $\Delta^{d-i}$ in $T$. If the link $L k\left(\Delta^{d-i}\right)$ of $\Delta^{d-i}$ in $T$ is not the boundary of any $i$-simplex of $T$, then the bistellar $i$-move on $\Delta^{d-i}$ consists in removing the join

$$
\Delta^{d-i} * \operatorname{Lk}\left(\Delta^{d-i}\right)
$$

from $T$ and replacing it with the join

$$
\partial \Delta^{d-i} * \Delta^{i}
$$

where $\Delta^{i}$ is a new $i$-simplex (not in $T$ before execution of the move) such that $\partial \Delta^{i}=L k\left(\Delta^{d-i}\right)$. In the case $d=3$ it is convenient to refer to a 0 -move as a $1 \rightarrow 4$ move, to a 1 -move as a $2 \rightarrow 3$ move, to a 2 -move as a $3 \rightarrow 2$ move, and to a 3 -move as a $4 \rightarrow 1$ move. See Figure 1 . Thus, a $1 \rightarrow 4$ move replaces one tetrahedron by four tetrahedra and a $2 \rightarrow 3$ move replaces two tetrahedra by three tetrahedra.

A $P L$ sphere is a simplicial complex that is piecewise linearly homeomorphic to the boundary of a simplex. A combinatorial manifold is a triangulation of a topological 


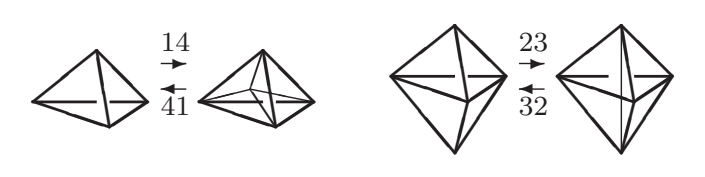

FIGURE 1. Pachner moves on a 3-manifold triangulation.

manifold such that the link at every vertex is a PL sphere. Two simplicial complexes triangulating a manifold are bistellarly equivalent if there exists a finite sequence of bistellar moves transforming one complex into the other.

Theorem 4.4. (Pachner.) Two combinatorial manifolds are bistellarly equivalent if and only if they are PL homeomorphic.

This result, together with Moise's work [Moise 52] asserting that every 3-manifold has a unique (up to PL homeomorphism) PL structure, implies that any two triangulations of a closed 3-manifold can be obtained from each other by executing a finite sequence of Pachner moves.

\section{PASSAGE TO QUARTIC GRAPHS}

Given any triangulation $T \in \operatorname{Triang}_{n}(M)$, there is an obvious way to associate a quartic graph $\mathrm{QG}(T)$ to $T$ such that combinatorial random walks on $T$ correspond to random walks on $\mathrm{QG}(T)$ : For $\mathrm{QG}(T)=(V, E)$, take the set of nodes $V$ to be the set of tetrahedra in $T$. Take the set of edges $E$ to be the set of 2-simplices in $T$, that is, $v \in V$ and $w \in V$ are joined by an edge iff the tetrahedra $v$ and $w$ share a common 2-face in $T$. Then $\mathrm{QG}(T)$ is indeed a quartic graph, and we have $m=2 n$. The combinatorial random walks of the introduction are precisely the discrete-time random walks of Section 3 on $\mathrm{QG}(T)$. The transition matrix is

$$
p_{v w}= \begin{cases}\frac{1}{4} & \text { if }(v, w) \text { is an edge } \\ 0 & \text { if not }\end{cases}
$$

and the stationary distribution is $\pi_{v}=1 / n$. We will be interested in the mean commute times between $v$ and $w$, $C_{\mathrm{QG}(T)}(v, w)$, and in the maximal mean commute time, $C(\mathrm{QG}(T))$, as defined in Sections 2 and 3.

The construction of $\mathrm{QG}(T)$ is related to the "facepairing graphs" of Burton [Burton 04], but there is an important difference: Burton triangulates by simplicial cell complexes, not by simplicial complexes in the strict sense. Consequently, his face-pairing graphs are 4 -valent multigraphs, i.e., may possess multiple edges and self-loops.

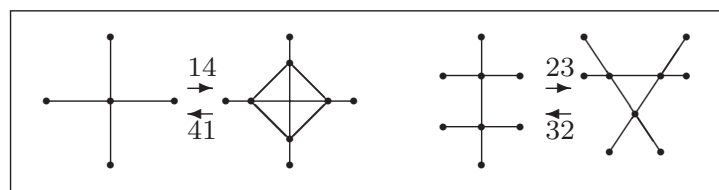

FIGURE 2. Pachner moves on quartic graphs.

For example, $S^{2} \times S^{1}$ can be triangulated by a simplicial cell complex containing two tetrahedra. The corresponding face-pairing graph has two nodes, each having one self-loop. The two nodes are joined by two distinct edges. Real projective space $\mathbb{R} P^{3}$ and the lens space $L(3,1)$ can also be triangulated by a cell complex with two tetrahedra. However, there are only two 4 -valent multigraphs on two nodes that can arise as face-pairing graphs, namely the graph described for $S^{2} \times S^{1}$, and the graph with four distinct edges between the two nodes. Thus two manifolds among $S^{2} \times S^{1}, \mathbb{R} P^{3}$, and $L(3,1)$ have the same face-pairing graph. In particular, stochastic processes running on this graph cannot distinguish between these two manifolds. This explains why we work with simplicial complexes, not cell complexes, in this paper.

Next, let us define the random variable whose investigation is the focus of this paper. We make $\operatorname{Triang}_{n}(M)$ into a probability space as follows: According to Lemma 4.3, $\operatorname{Triang}_{n}(M)$ is finite. We endow it with the uniform probability measure: For a triangulation $T \in$ $\operatorname{Triang}_{n}(M)$, set

$$
\mathbb{P}(\{T\})=\frac{1}{\left|\operatorname{Triang}_{n}(M)\right|} .
$$

Then the maximal mean commute time defines a random variable

$$
\begin{aligned}
C_{n}(M): \operatorname{Triang}_{n}(M) & \longrightarrow \mathbb{Q}, \\
T & \mapsto C(\mathrm{QG}(T)) .
\end{aligned}
$$

Sections 8.1 and 8.2 determine empirical density functions of $C_{n}(M)$ for $M=S^{3}, S^{2} \rtimes S^{1}, S^{2} \times S^{1}$, and $\mathbb{R} P^{3}$ for $n=27,40$. These values of $n$ are closely tied to the discrete geometry of the Klein bottle and projective space, respectively, and are thus of particular interest. Our software allows the investigation of any $n$, limited in practice only by time constraints and the available computing environment.

As discussed in the previous section, any two triangulations of $M$ are related by a finite sequence of Pachner moves. Figure 2 shows how the associated quartic graphs $\mathrm{QG}(T)$ of triangulations $T$ transform under the four Pachner moves. In fact, Pachner $1 \rightarrow 4$ moves in- 
duce a map

$$
\begin{gathered}
\{(G, v) \mid G \text { a quartic graph, } v \in V(G)\} \\
\longrightarrow\{\text { quartic graphs }\} .
\end{gathered}
$$

An analogous statement does not hold for $4 \rightarrow 1$ moves. For instance, applying a $4 \rightarrow 1$ move to any four nodes of the complete graph $K_{5}$ on five nodes leads to a multigraph with two nodes and four distinct edges between these two nodes. Furthermore, $2 \rightleftarrows 3$ moves do not readily give maps from quartic graphs to quartic graphs: the problem is that one cannot read off from the graph alone (not knowing the triangulation $T$ ) how to reconnect the edges to the rest of the graph.

\section{ELECTRICAL RESISTANCE}

To calculate mean commute times, we employ a reinterpretation in terms of resistance of electrical networks. Consider a graph $G$ as an electrical network in which each edge $(x, y)$ is a resistor of $R_{x y}$ ohms. Choose two distinct nodes $a$ and $b$ and apply voltage 1 at $a$ and ground $b$ (i.e., set voltage to 0 ). We are interested in determining, and giving a probabilistic interpretation of, the voltages $v_{x}$ that develop at nodes $x$, and the currents $i_{x y}$ flowing along the wires $(x, y)$ of the circuit. Let $i_{x}=\sum_{y} i_{x y}$ be the total current flowing from $x$. By Kirchhoff's node law, $i_{x}=0$ for $x \neq a, b$. Using Ohm's law

$$
i_{x y}=\frac{v_{x}-v_{y}}{R_{x y}}
$$

we deduce that

$$
v_{x}=\sum_{y} \frac{C_{x y}}{C_{x}} v_{y}
$$

where $C_{x y}=1 / R_{x y}$ is the conductance of the wire $(x, y)$ and $C_{x}=\sum_{y} C_{x y}$. Let us now specialize to $R_{x y}=1$ ohm for every edge. Then $C_{x y} / C_{x}=1 / d_{x}$, where $d_{x}$ is the degree of the node $x$. Using (3-2), we obtain, in terms of transition probabilities $p_{x y}$,

$$
v_{x}=\sum_{y} p_{x y} v_{y}, \quad x \neq a, b .
$$

This linear system, together with the boundary conditions $v_{a}=1, v_{b}=0$, has a unique solution. We define the effective resistance $\operatorname{er}_{G}(a, b)$ between $a$ and $b$ by

$$
\operatorname{er}_{G}(a, b)=\frac{v_{a}}{i_{a}}=\frac{1}{i_{a}} .
$$

Now the voltage can be interpreted as a hitting probability, since both functions are harmonic and they have the

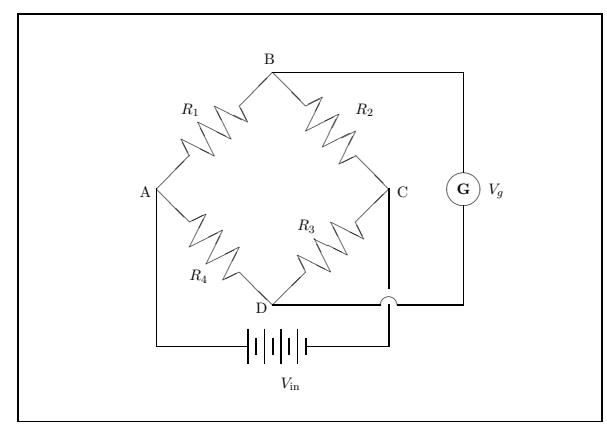

FIGURE 3. A Wheatstone bridge.

same boundary values. The current $i_{x y}$ flowing through the wire connecting $x$ to $y$ is proportional to the expected net number of times that a walking entity, starting at $a$ and walking until $b$ is reached, will move along the wire from $x$ to $y$. Chandra et al. [Chandra et al. 89] showed that the mean commute time is twice the number of edges times the effective resistance:

\section{Theorem 6.1. (Commute interpretation of resistance.)} Given two nodes $v, w$ in a graph $G$, the effective resistance $\operatorname{er}_{G}(v, w)$ between $v$ and $w$ is related to the mean commute time of the associated random walk by

$$
C_{G}(v, w)=2 m \cdot \operatorname{er}_{G}(v, w)
$$

where $m$ is the number of edges.

Note that if $G=\mathrm{QG}(T)$ for some $T \in \operatorname{Triang}_{n}(M)$, then

$$
C_{G}(v, w)=4 n \cdot \operatorname{er}_{G}(v, w)
$$

holds. This can be seen either graph-theoretically by noting that for a 4-regular graph, $m=2 n$ (see $(3-1)$ ), or topologically by observing that in a 3 -manifold the number of 2-simplices is twice the number of tetrahedra (see our discussion of $f$-vectors in Section 4 ). This means that within the set $\operatorname{Triang}_{n}(M)$, i.e., within the set of triangulations having $n$ tetrahedra, the commute times are proportional to the electrical resistance values. We shall write $\operatorname{er}(G)$ for the maximal effective resistance of a graph $G$,

$$
\operatorname{er}(G)=\max _{v, w} \operatorname{er}_{G}(v, w)
$$

It is perhaps interesting to note that performing a Pachner $1 \rightarrow 4$ move on a node $v$ of $\mathrm{QG}(T)$ (see Figure 2 ) corresponds physically to removing $v$ and replacing it with an electrical network component called a Wheatstone bridge; see Figure 3. 
The Wheatstone bridge is a circuit used for precise measurements of resistance. It consists of a source of electrical current (such as a battery) and a galvanometer that connects two parallel branches of a diamond containing four resistors, three of which are known. In order to determine the resistance of the unknown resistor, the resistances of the other three are adjusted and balanced until the current passing through the galvanometer decreases to zero.

Definition 6.2. Let $M^{3}$ be a closed 3-manifold and $T \in \operatorname{Triang}_{n}(M)$ a triangulation. The (maximal effective) electrical resistance $\operatorname{er}(T)$ of $T$ is given by

$$
\operatorname{er}(T)=\operatorname{er}(\mathrm{QG}(T))
$$

The (maximal mean) commute time $C(T)$ of $T$ is given by

$$
C(T)=C(\mathrm{QG}(T))
$$

Let $n_{\min }$ be the unique natural number such that

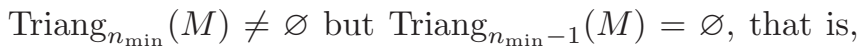
$n_{\text {min }}$ is the smallest number of tetrahedra necessary to triangulate $M$. The electrical resistance $\operatorname{er}(M)$ of the manifold $M$ is defined to be

$$
\operatorname{er}(M)=\min \left\{\operatorname{er}(T) \mid T \in \operatorname{Triang}_{n_{\min }}(M)\right\}
$$

By definition, $\operatorname{er}(M)$ is a topological invariant of $M$.

Example 6.3. We compute $\operatorname{er}\left(S^{3}\right)$. The unique minimal triangulation $T$ of the sphere is the boundary complex of a standard 4-dimensional simplex. Explicitly, $T$ has vertices $\{1,2,3,4,5\}$ and five tetrahedra

$$
(1,2,3,4), \quad(1,2,3,5), \quad(1,2,4,5), \quad(1,3,4,5), \quad(2,3,4,5)
$$

Therefore,

$$
\mathrm{QG}(T)=K_{5}
$$

the complete graph on five nodes. By Foster's identity,

$$
\sum_{(v, w) \in E} \operatorname{er}(v, w)=n-1=4
$$

By the symmetry of the complete graph, the er $(v, w)$ are all equal. Since there are 10 edges, we see that

$$
\operatorname{er}\left(K_{5}\right)=\frac{2}{5}
$$

whence $\operatorname{er}\left(S^{3}\right)=\frac{2}{5}$. (Alternatively, one may solve the linear system directly for $K_{5}$, or one argues on probabilistic grounds that the maximal mean commute time for the complete graph $K_{n}$ is $C\left(K_{n}\right)=2(n-1)$, so that by Theorem 6.1, $\operatorname{er}\left(K_{n}\right)=(n-1) / 2 n$.) Moreover, if $M$ is any manifold, then $\operatorname{er}(M) \geq \frac{2}{5}$ : By a result of [Coppersmith et al. 96], the effective resistance between any two nodes $v, w$ of a simple, connected graph $G$ satisfies the lower bound

$$
\operatorname{er}_{G}(v, w) \geq \frac{1}{d_{v}+1}+\frac{1}{d_{w}+1},
$$

where $d_{v}, d_{w}$ are the degrees of $v, w$, respectively. For any triangulation $T$ of $M, G=\mathrm{QG}(T)$ is quartic, and thus $d_{v}=d_{w}=4$ for all $v, w$.

The following table records the electrical resistances of some 3-manifolds:

\begin{tabular}{|c|l|}
\hline$M$ & $\operatorname{er}(M)$ \\
\hline$S^{3}$ & 0.4 \\
$\mathbb{R} P^{3}$ & $0.7464(*)$ \\
$L(3,1)$ & $0.7621(*)$ \\
$S^{2} \rtimes S^{1}$ & 0.8056 \\
\hline
\end{tabular}

The values marked by $(*)$ are known to be upper bounds, but the reverse inequalities rely on assuming the validity of two conjectures in discrete geometry. The conjectures say that Walkup's triangulation of $\mathbb{R} P^{3}$ with $f$-vector $(11,51,80,40)$ [Walkup 70] is unique among all triangulations of $\mathbb{R} P^{3}$ with this $f$-vector, and that Brehm's triangulation of $L(3,1)$ with $f$-vector $(12,66,108,54)$ is unique among vertex minimal triangulations; see [Lutz 05].

One may think of the electrical resistance of a 3manifold as a refined complexity measure with values in the rational numbers. For example, there are three manifolds with Matveev complexity 0 , namely $S^{3}, \mathbb{R} P^{3}$, and $L(3,1)$. As the above table shows, these are further distinguished by their electrical resistance values.

\section{THE ALGORITHM}

Let us describe the algorithm used to produce the experimental data. The input consists of a quintuple $(T, n, s, l, N)$, where $T$ is a seed triangulation of a 3 manifold $M$ containing $n_{0}$ tetrahedra, $n \geq n_{0}$ is the desired number of tetrahedra of the random triangulations of $M$ to be generated, $s$ is the desired sample size, and $l, N \geq 0$ are parameters that control the generation of the random triangulations. We refer to $l$ as the excess Pachner length and to $N$ as the Markov length. Given such a quintuple, the algorithm proceeds roughly as follows: It applies random Pachner forward moves, i.e., $1 \rightarrow 4$ and 
$2 \rightarrow 3$ moves, starting out from $T$, until a triangulation with $n+l$ tetrahedra is reached. Once $n+l$ tetrahedra have been reached, random reverse Pachner moves, i.e., $4 \rightarrow 1$ and $3 \rightarrow 2$ moves, are applied until a triangulation $T_{1}$ with $n$ tetrahedra is reached. Then $T_{1}$ is again inflated to $n+l$ tetrahedra, and reduced to $n$ tetrahedra, yielding a triangulation $T_{2}$, etc., until one arrives after $N$ steps at a triangulation $T_{N}$. The algorithm then generates the associated quartic graph $\mathrm{QG}\left(T_{N}\right)$ and computes its maximal electrical resistance using the method of Section 6. This amounts to solving a large linear system, which is done numerically by Jacobi iteration. From the resistance, the commute time $C\left(T_{N}\right)$ is computed using Theorem 6.1. This entire process is carried out $s$ times, and each time a density curve is updated. For the seed triangulation $T$, we typically start with a vertex minimal triangulation for a given manifold. ${ }^{3}$ The output of the algorithm consists of the densities for electrical resistance and maximal mean commute times, as well as their sample means. We have implemented this algorithm in $\mathrm{C}$ for speed. The high-quality FSU-ULTRA random-number generator by Marsaglia and Zaman has been used [Marsaglia and Zaman 91]. ${ }^{4}$

In the following section, we describe the results, as well as their implications, of executing the algorithm for $n=27$ and $n=40$. We chose these two values because of their distinguished roles in discrete geometry: $n=27$ is closely tied to the 3-dimensional Klein bottle, since it is the minimal number of tetrahedra required to triangulate the Klein bottle; $n=40$ is closely tied to real projective space, since it is the minimal number of tetrahedra required to triangulate $\mathbb{R} P^{3}$.

The empirical densities presented here are based on a sample size of $s=3000$ triangulations. Experiments show that this sample size is adequate for our purposes, i.e., drawing multiple random samples of 3000 leads only to small changes in the density curves. An excess Pachner length of $l=35$ and a Markov length of $N=5$ were employed.

In what sense and why does this algorithm generate roughly uniform triangulation samples? We shall first define abstractly a class of stratified graphs, for which we isolate general conditions under which the algorithm provably generates the uniform probability measure in the limit (Theorem 7.7). We will then create a stratified

\footnotetext{
${ }^{3}$ These can be found, e.g., at the manifold page of F. Lutz, http://www.math.tu-berlin.de/diskregeom/stellar/.

${ }^{4}$ The code is available at http://www.mathi.uni-heidelberg.de/ $\sim$ banagl/statisticaltopology/er.c.
}

model of the Pachner graph and discuss how it fits into the abstract framework.

Let us recall the definition of a multipartite graph:

Definition 7.1. A multipartite graph is a graph $G=$ $(V, E)$ together with a partition $V=\bigcup_{i \in I} V_{i}$ of the set of nodes ( $I$ some index set) such that no two nodes in the same $V_{i}$ are joined by an edge in $E$.

A special class of multipartite graphs is that of the stratified graphs:

Definition 7.2. A stratified graph is a multipartite graph $G=(V, E)$ with node partition $V=V_{0} \cup V_{1} \cup V_{2} \cup \cdots$ such that if $v \in V_{i}, w \in V_{j}, i<j$, and $(v, w) \in E$ is an edge, then $j=i+1$. (In other words, edges exist only between adjacent $V_{i}$.) The $V_{i}$ are called the strata of $G$. Note that in a stratified graph, the edges starting at a node $v \in V_{i}$ can be classified into forward edges (if they end in $V_{i+1}$ ) and backward edges (if they end in $\left.V_{i-1}\right)$. The degree $d_{v}$ of $v$ is the sum of the forward degree $d_{v}^{+}$(the number of forward edges emanating from $v$ ) and the backward degree $d_{v}^{-}$(the number of backward edges emanating from $v$ ).

The following three conditions on a stratified graph will be the hypotheses of the uniform measure theorem below.

Definition 7.3. A stratified graph is forward connected if for every $i=0,1,2, \ldots$ and any two nodes $v, v^{\prime} \in V_{i}$, there exists a node $w \in V_{i+l}$ for some $l>0$ such that both $v$ and $v^{\prime}$ can be connected to $w$ by paths that use only forward edges.

Definition 7.4. A stratified graph is stratum-regular if there exist sequences $\left(d_{0}^{+}, d_{1}^{+}, d_{2}^{+}, \ldots\right)$ and $\left(d_{0}^{-}=\right.$ $\left.0, d_{1}^{-}, d_{2}^{-}, \ldots\right)$ of positive (except for $d_{0}^{-}$) integers such that for every $i=0,1,2, \ldots$ and every $v \in V_{i}$, one has $d_{v}^{+}=d_{i}^{+}, d_{v}^{-}=d_{i}^{-}$. In other words, the forward degrees are constant on every stratum, and the backward degrees are constant on every stratum.

Definition 7.5. A stratified graph is stratum-finite if every stratum $V_{i}$ is a finite set.

Let $G$ be a forward-connected, stratum-finite, stratum-regular, stratified graph; let $v_{0} \in V_{0}$ be any node; and let $L, N$ be two positive integers. Consider 
the following random algorithm operating on the input quadruple $\left(G, v_{0}, L, N\right)$ :

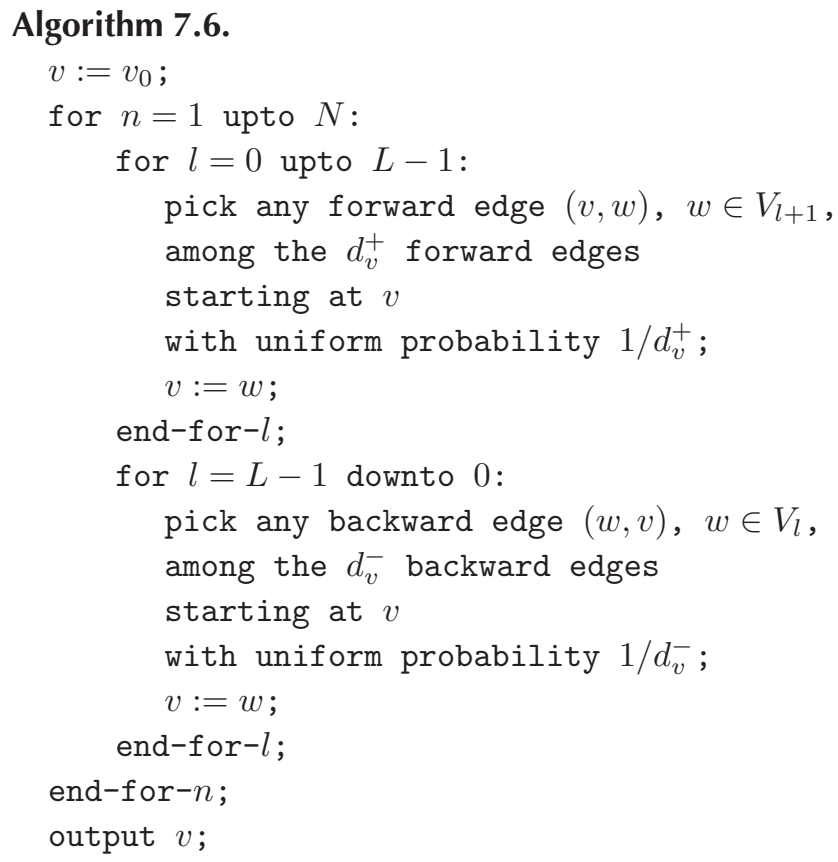

Theorem 7.7. Let $G$ be a forward-connected, stratumfinite, stratum-regular, stratified graph. Then there exists a positive integer $L$ such that for any $v_{0} \in V_{0}$, Algorithm 7.6 draws nodes from $V_{0}$ with uniform probability $1 /\left|V_{0}\right|$ as $N \rightarrow \infty$.

Proof: The first step is to find $L$. Let $v, v^{\prime} \in V_{0}$ be nodes in the 0-stratum. Since $G$ is forward connected, there exists a node $w \in V_{l\left(v, v^{\prime}\right)}$ for some $l\left(v, v^{\prime}\right)>0$ such that both $v$ and $v^{\prime}$ can be connected to $w$ by paths that use only forward edges. Set

$$
L=\max _{v, v^{\prime} \in V_{0}} l\left(v, v^{\prime}\right)
$$

This maximum exists because $G$ is stratum-finite.

The second step is to build a Markov chain that describes the action of Algorithm 7.6. The states are given by

$$
V_{0} \cup V_{1}^{+} \cup V_{1}^{-} \cup V_{2}^{+} \cup V_{2}^{-} \cup \cdots \cup V_{L-1}^{+} \cup V_{L-1}^{-} \cup V_{L},
$$

where $V_{k}^{+}$and $V_{k}^{-}, k=1, \ldots, L-1$, are two disjoint copies of the set $V_{k}$. For each $k=0, \ldots, L$, choose a bijection between $V_{k}$ and the set $\left\{1,2, \ldots,\left|V_{k}\right|\right\}$. This relabeling allows us to refer to nodes of $V_{k}$ by natural numbers $i, 1 \leq i \leq\left|V_{k}\right|$. The transition matrix $P$ has the following form:

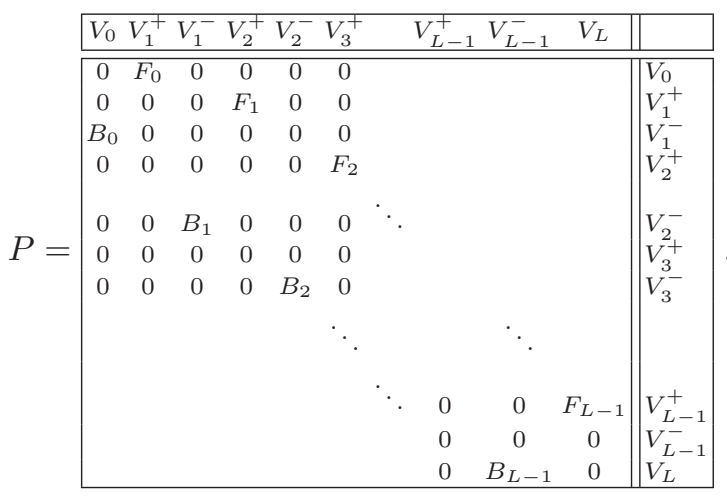

The $F_{k}$ are matrices of order $\left|V_{k}\right| \times\left|V_{k+1}\right|$ that contain the probabilities $f_{i j}^{k}$ of going from node $i \in V_{k}$ to node $j \in V_{k+1}$ via a forward edge. Since $G$ is stratum-regular, $f_{i j}^{k}= \begin{cases}1 / d_{k}^{+}, & \text {if }(i, j) \text { is a forward edge starting at } i, \\ 0, & \text { otherwise. }\end{cases}$ Since $F_{k}$ is a probability matrix, we have

$$
\sum_{j} f_{i j}^{k}=1
$$

for every $i \in V_{k}$. The $B_{k}$ are matrices of order $\left|V_{k+1}\right| \times$ $\left|V_{k}\right|$ that contain the probabilities $b_{i j}^{k}$ of going from node $i \in V_{k+1}$ to node $j \in V_{k}$ via a backward edge. Again, since $G$ is stratum-regular,

$b_{i j}^{k}= \begin{cases}1 / d_{k+1}^{-}, & \text {if }(j, i) \text { is a backward edge starting at } i, \\ 0, & \text { otherwise. }\end{cases}$

On the other hand, the backward transition probabilities can be computed from the forward probabilities:

$$
B_{k}=\operatorname{diag}\left(\frac{1}{\sum_{i} f_{i 1}^{k}}, \ldots, \frac{1}{\sum_{i} f_{i\left|V_{k+1}\right|}^{k}}\right) \cdot F_{k}^{T},
$$

where $F_{k}^{T}$ denotes the transpose of $F_{k}$. Let $i \in V_{k+1}$. There exists a $j \in V_{k}$ such that $(j, i)$ is a backward edge starting at $i$, because the backward degree of node $i$ is $d_{k+1}^{-}>0$. For this $j$,

$$
\frac{1}{d_{k+1}^{-}}=b_{i j}^{k}=\frac{1}{\sum_{l} f_{l i}^{k}} \cdot f_{j i}^{k}=\frac{1}{\sum_{l} f_{l i}^{k}} \cdot \frac{1}{d_{k}^{+}} .
$$

Thus the sum

$$
\sum_{l} f_{l i}^{k}=\frac{d_{k+1}^{-}}{d_{k}^{+}}
$$

is independent of the node $i$. Let $Q$ be the $V_{0} \times V_{0}$ block of the power $P^{2 L}$. To establish the statement of the theorem, we must show that

$$
\lim _{N \rightarrow \infty} Q^{N}
$$


exists and that all of its entries are equal to $\frac{1}{m}$, where $m=\left|V_{0}\right|$.

In order to show that the limit exists, we shall show first that $Q=\left(q_{v v^{\prime}}\right)_{v, v^{\prime} \in V_{0}}$ has only positive entries (in particular, the Markov chain defined by $Q$ is a regular Markov chain): Let $v, v^{\prime} \in V_{0}$ be nodes in the 0 stratum. Since $G$ is forward connected, there exists a node $w \in V_{l\left(v, v^{\prime}\right)}$ such that $v$ and $v^{\prime}$ can be connected to $w$ by paths $\gamma$ and $\gamma^{\prime}$, respectively, that use only forward edges. Construct a path from $v$ to $v^{\prime}$ with exactly $2 L$ steps as follows: First go from $v$ to $w$ using $\gamma$. Note that $l\left(v, v^{\prime}\right) \leq L$. If $l\left(v, v^{\prime}\right)=L$, then return from $w$ to $v^{\prime}$ going backward along $\gamma^{\prime}$. If $l\left(v, v^{\prime}\right)<L$, select any forward edge starting at $w$, leading to some node $w_{1} \in V_{l\left(v, v^{\prime}\right)+1}$. Continue to select nodes $w_{2}, w_{3}, \ldots$ this way, until you reach a node $w_{L-l\left(v, v^{\prime}\right)} \in V_{L}$. Then

$\gamma w_{1} w_{2} \ldots w_{L-l\left(v, v^{\prime}\right)-1} w_{L-l\left(v, v^{\prime}\right)} w_{L-l\left(v, v^{\prime}\right)-1} \ldots w_{2} w_{1} \gamma_{\text {rev }}^{\prime}$

is the required path. (Here, $\gamma_{\text {rev }}^{\prime}$ means $\gamma^{\prime}$ in reverse order.) Since there is a path going from $v$ to $v^{\prime}$ in exactly $2 L$ steps, the probability $q_{v v^{\prime}}$ of going from $v$ to $v^{\prime}$ in $2 L$ steps is positive. Now the fundamental limit theorem for regular Markov chains asserts that the limit

$$
\lim _{N \rightarrow \infty} Q^{N}=\Pi
$$

exists, and that all rows of $\Pi$ are equal.

Lastly, we show that every row $\pi$ of $\Pi$ equals the uniform vector $u=\left(\frac{1}{m}, \frac{1}{m}, \ldots, \frac{1}{m}\right)$. The row $\pi$ is the unique fixed probability vector determined by the equation

$$
\pi Q=\pi
$$

Hence it suffices to show that the equation

$$
u Q=u
$$

holds. The matrix $Q$ factors as

$$
Q=F_{0} F_{1} \cdots F_{L-1} B_{L-1} \cdots B_{1} B_{0} .
$$

We have

$$
\begin{aligned}
u F_{0} & =\left(\frac{1}{m} \sum_{i} f_{i 1}^{0}, \ldots, \frac{1}{m} \sum_{i} f_{i\left|V_{1}\right|}^{0}\right) \\
& =\left(\frac{1}{m} \frac{d_{1}^{-}}{d_{0}^{+}}, \ldots, \frac{1}{m} \frac{d_{1}^{-}}{d_{0}^{+}}\right)
\end{aligned}
$$

using (7-2), and inductively

$$
u F_{0} F_{1} \cdots F_{L-1}=\frac{1}{m} \frac{d_{1}^{-}}{d_{0}^{+}} \frac{d_{2}^{-}}{d_{1}^{+}} \cdots \frac{d_{L}^{-}}{d_{L-1}^{+}}(1, \ldots, 1) .
$$

At this point, one takes the first reverse step, yielding the distribution

$$
\begin{aligned}
u F_{0} & F_{1} \cdots F_{L-1} B_{L-1} \\
= & u F_{0} F_{1} \cdots F_{L-1} \\
& \times \operatorname{diag}\left(\frac{1}{\sum_{i} f_{i 1}^{L-1}}, \ldots, \frac{1}{\sum_{i} f_{i\left|V_{L}\right|}^{L-1}}\right) \cdot F_{L-1}^{T} \\
= & u F_{0} F_{1} \cdots F_{L-1} \operatorname{diag}\left(\frac{d_{L-1}^{+}}{d_{L}^{-}}, \ldots, \frac{d_{L-1}^{+}}{d_{L}^{-}}\right) \cdot F_{L-1}^{T} \\
= & \frac{1}{m} \frac{d_{1}^{-}}{d_{0}^{+}} \frac{d_{2}^{-}}{d_{1}^{+}} \cdots \frac{d_{L-1}^{-}}{d_{L-2}^{+}}(1, \ldots, 1) \cdot F_{L-1}^{T} \\
= & \frac{1}{m} \frac{d_{1}^{-}}{d_{0}^{+}} \frac{d_{2}^{-}}{d_{1}^{+}} \cdots \frac{d_{L-1}^{-}}{d_{L-2}^{+}}\left(\sum_{j} f_{1 j}^{L-1}, \ldots, \sum_{j} f_{\left|V_{L}\right| j}^{L-1}\right) \\
= & \frac{1}{m} \frac{d_{1}^{-}}{d_{0}^{+}} \frac{d_{2}^{-}}{d_{1}^{+}} \cdots \frac{d_{L-1}^{-}}{d_{L-2}^{+}}(1, \ldots, 1),
\end{aligned}
$$

using (7-1). Thus, inductively,

$$
u Q=u F_{0} F_{1} \cdots F_{L-1} B_{L-1} \cdots B_{1} B_{0}=\frac{1}{m}(1, \ldots, 1)=u .
$$

Let $M^{3}$ be a closed 3-manifold. The Pachner graph $P(M)$ of $M$ (sometimes called the "bistellar flip graph") is defined as follows. Its set of nodes consists of the isomorphism classes of triangulations of $M$, that is, its set of nodes is $\bigcup_{n} \operatorname{Triang}_{n}(M)$. Two triangulations are joined by an edge iff there is a Pachner move transforming one into the other. In the following, we shall use the shorthand notation $\operatorname{Tr}_{n}=\operatorname{Triang}_{n}(M)$. Given a triangulation $T$ of $M$ with $n$ tetrahedra, one can select any one of these tetrahedra and perform a $1 \rightarrow 4$ move on it. Thus, in order to create a rough model of the Pachner graph, we will assume that the number $d_{n, 14}$ of $1 \rightarrow 4$ edges emanating from a triangulation in $\operatorname{Tr}_{n}$ is proportional to $n$. Similarly, we will assume that the numbers $d_{n, 23}, d_{n, 41}$, and $d_{n, 32}$ of $2 \rightarrow 3,4 \rightarrow 1$, and $3 \rightarrow 2$ edges, respectively, are proportional to $n$, say $d_{n, s}=\lambda_{s} n, s \in\{14,41,23,32\}$.

This type of regularity assumption is further motivated by considering the symmetries of triangulations. If there were substantial irregularity over $\operatorname{Tr}_{n}$, then this would mean that a substantial fraction of triangulations $T$ in $\operatorname{Tr}_{n}$ have the property that most, say, $1 \rightarrow 4$ moves on $T$ lead to the same isomorphism class of triangulations in $\operatorname{Tr}_{n+3}$. This in turn would mean that a substantial fraction of triangulations in $\operatorname{Tr}_{n}$ are highly symmetric, i.e., Aut $(T)$ is large. Suppose, for example, that $\phi \in \operatorname{Aut}(T)$ and that $\phi$ maps a tetrahedron $t_{1}$ in $T$ to 
some other tetrahedron $t_{2}$. Then $\phi$ can be extended to yield an isomorphism from the result of a $1 \rightarrow 4$ move on $t_{1}$ to the result of a $1 \rightarrow 4$ move on $t_{2}$. Now in fact, however, this ubiquity of large automorphism groups cannot be observed in practice. Even distinguished triangulations, such as minimal ones, typically have small automorphism groups.

To investigate $\operatorname{Aut}(T)$, one observes that if a combinatorial manifold has a combinatorial symmetry $\phi$, then the links of a vertex $v$ and its image $\phi(v)$ must be combinatorially equivalent. For instance, the only known triangulation $T$ of $L(3,1)$ with $n=54$ has 12 vertices, 6 of which have Altshuler-Steinberg invariant 134784, 3 of which have Altshuler-Steinberg invariant 133056, and the remaining 3 have Altshuler-Steinberg invariant 112320. Thus Aut $(T)$ is a subgroup of $S_{6} \times S_{3} \times S_{3}$. However, most of these symmetries cannot be realized and the actual automorphism group is $S_{3}$.

Naturally, the above degrees are related: The number of edges between $\operatorname{Tr}_{n}$ and $\operatorname{Tr}_{n+1}$ is equal to both $\left|\operatorname{Tr}_{n}\right|$. $d_{n, 23}$ and $\left|\operatorname{Tr}_{n+1}\right| \cdot d_{n+1,32}$. The number of edges between $\operatorname{Tr}_{n}$ and $\operatorname{Tr}_{n+3}$ is equal to both $\left|\operatorname{Tr}_{n}\right| \cdot d_{n, 14}$ and $\left|\operatorname{Tr}_{n+3}\right|$. $d_{n+3,41}$. Thus

$$
\frac{d_{n, 14}}{d_{n+3,41}}=\frac{d_{n+2,23}}{d_{n+3,32}} \frac{d_{n+1,23}}{d_{n+2,32}} \frac{d_{n, 23}}{d_{n+1,32}},
$$

which implies

$$
\frac{\lambda_{14}}{\lambda_{41}}=\left(\frac{\lambda_{23}}{\lambda_{32}}\right)^{3}
$$

for the proportionality constants. Note that $\lambda_{14}>\lambda_{41}$, since after selecting one tetrahedron, one can immediately perform a $1 \rightarrow 4$ move, but one must select three neighboring tetrahedra such that all four tetrahedra satisfy a certain condition (stated in Section 4) before one can perform a $4 \rightarrow 1$ move. Then (7-3) implies that $\lambda_{23}>\lambda_{32}$ as well, which is consistent with experimental observations. Note also that our model implies that the size of $\operatorname{Tr}_{n}$ grows essentially exponentially with $n$. For example, in terms of $2 \leftrightarrow 3$ moves,

$$
\left|\operatorname{Tr}_{N+k}\right|=\left|\operatorname{Tr}_{N}\right| \frac{N}{N+k}\left(\frac{\lambda_{23}}{\lambda_{32}}\right)^{k}
$$

Essentially exponential growth is indeed suggested by results available in dimension 2: Tutte [Tutte 62] proves that the number of nonisomorphic triangulations of a triangle is asymptotically equal to

$$
\frac{1}{16}\left(\frac{3}{2 \pi}\right)^{1 / 2} k^{-5 / 2}\left(\frac{256}{27}\right)^{k+1}
$$

where $3 k=r-6$ and $r$ is the number of internal edges.

In order to stratify $P(M)$, it would be most natural to set $V_{n}=\operatorname{Tr}_{n}$. While $P(M)$ would thus be multipartite, it would, however, not be stratified because a $1 \rightarrow 4$ move connects $V_{n}$ to $V_{n+3}$ and not to $V_{n+1}$ as required. Fix a number $N$ of tetrahedra. If we are interested in investigating triangulations of $M$ with at least $N$ tetrahedra, then we may associate to $P(M)$ a stratified graph $\mathrm{SP}_{N}(M)=(V, E)$ in a natural way as follows: For $k \geq 0$, set

$$
V_{N+k}=\operatorname{Tr}_{N+k} \cup \cdots \cup \operatorname{Tr}_{N+3 k+2}
$$

and

$$
V=\bigcup_{k \geq 0} V_{N+k}
$$

(These unions are to be disjoint.) For $T, T^{\prime} \in V,\left(T, T^{\prime}\right)$ is an edge in $E$ iff $T \in \operatorname{Tr}_{n} \subset V_{N+k}$, for some $n$ and $k$, and either $T^{\prime} \in \operatorname{Tr}_{n+1} \subset V_{N+k+1}$ and there is a $2 \rightarrow 3$ move transforming $T$ into $T^{\prime}$, or $T^{\prime} \in \operatorname{Tr}_{n+3} \subset V_{N+k+1}$ and there is a $1 \rightarrow 4$ move transforming $T$ into $T^{\prime}$. Then $\mathrm{SP}_{N}(M)$ is a stratified graph. (We do not reindex so that $V_{N}$ becomes $V_{0}$.) Lemma 4.3 implies that $\operatorname{SP}_{N}(M)$ is stratum-finite. Moreover, one expects $\mathrm{SP}_{N}(M)$ to be forward connected for the following reason: a PL structure on a manifold is a class of locally finite triangulations that is closed under linear subdivision and such that any two triangulations in it have a common subdivision. A 3-manifold has a unique PL structure. Thus any two triangulations of a 3-manifold have a common subdivision. It is a classic conjecture in topology that if two complexes have isomorphic subdivisions, then they have isomorphic subdivisions each obtained by a sequence of stellar subdivisions (with no welds being used). See [Lickorish 99, p. 311]. Thus the subdivisions can be reached using forward Pachner moves only. Finally, our model of the Pachner graph implies that $\operatorname{SP}_{N}(M)$ is approximately stratum-regular: on $\operatorname{Tr}_{n} \subset V_{N+k}$, the forward degree is $\left(\lambda_{14}+\lambda_{23}\right) n$, whence on $V_{N+k}$ the forward degree varies between $\left(\lambda_{14}+\lambda_{23}\right)(N+k)$ and $\left(\lambda_{14}+\lambda_{23}\right)(N+3 k+2)$. Let $\mu_{k}$ and $\sigma_{k}^{2}$ denote mean and variance, respectively, of the forward degree over $V_{N+k}$. Neglecting the term $N /(N+k)$ in $(7-4)$, we have

$$
\mu_{k} \approx \int_{\lambda(N+k)}^{\lambda(N+3 k+2)} x s(x) \mathrm{d} x / \int_{\lambda(N+k)}^{\lambda(N+3 k+2)} s(x) \mathrm{d} x,
$$

where

$$
\begin{aligned}
& \lambda=\lambda_{14}+\lambda_{23}, \quad s(x)=\left|\operatorname{Tr}_{N}\right| e^{\alpha x}, \\
& \alpha=\log \left(\frac{\lambda_{23}}{\lambda_{32}}\right)>0 .
\end{aligned}
$$


It follows that

$$
\lambda(N+3 k+2)-\mu_{k} \longrightarrow \frac{1}{\alpha}
$$

as $k \rightarrow \infty$. Using the approximation

$$
\sigma_{k}^{2} \approx \frac{\int_{\lambda(N+k)}^{\lambda(N+3 k+2)}\left(x-\mu_{k}\right)^{2} s(x) \mathrm{d} x}{\int_{\lambda(N+k)}^{\lambda(N+3 k+2)} s(x) \mathrm{d} x},
$$

and writing

$$
\mu_{k}=\lambda(N+3 k+2)-\frac{1}{\alpha}+\epsilon_{k}, \quad \epsilon_{k} \rightarrow 0,
$$

one obtains

$$
\sigma_{k}^{2} \longrightarrow \frac{1}{\alpha^{2}}=\frac{1}{\log ^{2}\left(\frac{\lambda_{23}}{\lambda_{32}}\right)}
$$

as $k \rightarrow \infty$. In particular, the ratio of the variance of the forward degree over $V_{N+k}$ to the forward degrees themselves goes to zero as $k \rightarrow \infty$ and in this sense $\operatorname{SP}_{N}(M)$ is roughly stratum-regular. Then, according to Theorem 7.7 applied to $\mathrm{SP}_{N}(M)$, Algorithm 7.6 constructs elements of $V_{N}$ uniformly.

There exist, of course, other methods for constructing random triangulations. In dimension 2, Leibon [Leibon 99] uses a procedure having its origin in Delaunay's "empty sphere" method [Delaunay 28] to obtain geodesic triangulations of a surface $\Sigma^{2}$ equipped with a Riemannian metric $g$. Let $\delta>0$ be a number with $\delta<\frac{i}{6}$, where $i$ is the injectivity radius of $\Sigma^{2}$. Given a set $P=\left\{p_{1}, \ldots, p_{n}\right\}$ of points on $\Sigma^{2}$, put in a face for a triple of these points or an edge for a pair of these points if the triple or pair lies on a disk of radius less than $\delta$ that contains none of $p_{1}, \ldots, p_{n}$ in its interior. This yields an abstract simplicial complex $K_{P}$, together with a map $R:\left|K_{P}\right| \rightarrow \Sigma^{2}$. Call $P \delta$-dense if each open $\delta$-ball contains at least one $p_{j}$ and $P$ has no four of its points on a circle of radius less than $\min \left(\frac{i}{6}, \tau\right)$, where $\tau$ is the strong convexity radius of $\Sigma^{2}$. Leibon proves that if $\delta<\min \left(\frac{i}{6}, \tau\right)$ and $P$ is $\delta$-dense, then $R$ is a homeomorphism. In order to construct random triangulations of $\Sigma^{2}$, Leibon puts random sets of points on $\Sigma^{2}$ distributed via a Poisson distribution relative to $g$. (He applies this, for example, in giving a probabilistic proof of the GaussBonnet theorem.) In dimension 3, this method is not as canonical as in dimension 2, since in dimension 3 there are no natural geometric simplices and faces.

\section{EXPERIMENTAL RESULTS}

\subsection{The 3-Dimensional Klein Bottle}

The empirical density of the random variable $C_{27}\left(S^{3}\right)$ (i.e., maximal mean commute time of random walks on triangulations of $S^{3}$ with 27 tetrahedra) is shown in Figure 4. Its sample mean is

$$
\bar{C}_{27}\left(S^{3}\right)=139.9 \text {. }
$$

Theorem 8.1. Suppose an unknown manifold $M$ is triangulated with 27 tetrahedra. If the maximal mean commute time associated with the combinatorial random walk on this triangulation is greater than or equal to 88, then $M$ is the 3-sphere.

Proof: Discrete geometry implies that when $n=27, M$ has to be $S^{3}$ or $S^{2} \rtimes S^{1}$. In fact, let us recall Theorem 4.1: With the exception of $S^{3}, S^{2} \rtimes S^{1}$, and $S^{2} \times S^{1}$, which have minimal triangulations with 5, 9, and 10 vertices, respectively, every other triangulated 3-manifold has at least 11 vertices. Now if $n=f_{3}=27$, then $f_{1}-f_{0}=27$ by (4-1). Walkup's inequality (Theorem 4.2) states that

$$
f_{1} \geq 4 f_{0}+\gamma(M)
$$

If $M$ is not $S^{3}$, then $\gamma(M) \geq 0$. Thus $27 \geq 3 f_{0}$, whence $f_{0} \leq 9$. Hence $M$ must be $S^{2} \rtimes S^{1}$.

Theorems 4.1 and 4.2 imply that any triangulation $T \in \operatorname{Triang}_{27}\left(S^{2} \rtimes S^{1}\right)$ has $f_{0}=9$, and consequently has $f$-vector $f=(9,36,54,27)$. By [Altshuler and Steinberg 76] there is only one triangulation of $S^{2} \rtimes S^{1}$ with this $f$ vector. This unique triangulation $T \in \operatorname{Triang}_{27}\left(S^{2} \rtimes S^{1}\right)$ has

$$
\operatorname{er}(T)=0.8056, \quad C_{27}(T)=87.01 .
$$

Thus, if $C_{27} \geq 88$, then $M$ is $S^{3}$.

Heuristic 8.2. Suppose an unknown manifold $M$ is triangulated with 27 tetrahedra. Assume we know that the maximal mean commute time associated with the combinatorial random walk on this triangulation is less than or equal to 102 . Then $M$ is either the 3 -sphere or $S^{2} \rtimes S^{1}$

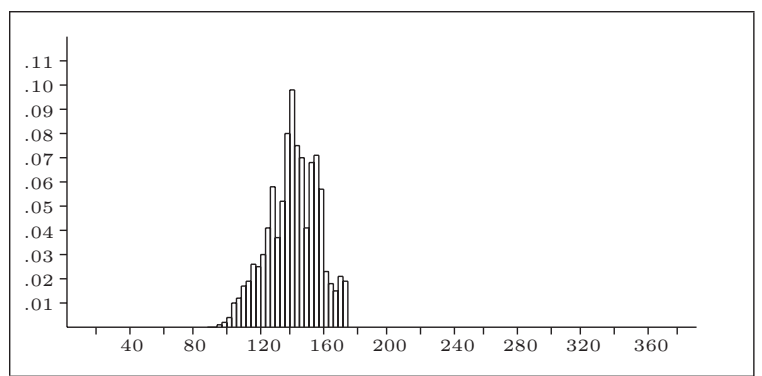

FIGURE 4. Commute time density on the 3-sphere with 27 tetrahedra. 
with probabilities

$$
\begin{aligned}
\mathbb{P}\left(S^{3} \mid C \leq 102\right) & =\frac{0.009 \mathbb{P}\left(S^{3}\right)}{0.009 \mathbb{P}\left(S^{3}\right)+\mathbb{P}\left(S^{2} \rtimes S^{1}\right)}, \\
\mathbb{P}\left(S^{2} \rtimes S^{1} \mid C \leq 102\right) & =\frac{\mathbb{P}\left(S^{2} \rtimes S^{1}\right)}{0.009 \mathbb{P}\left(S^{3}\right)+\mathbb{P}\left(S^{2} \rtimes S^{1}\right)} .
\end{aligned}
$$

Verification of Heuristic 8.2: Let

$$
\begin{aligned}
& U=\operatorname{Triang}_{27}\left(S^{3}\right), \\
& V=\operatorname{Triang}_{27}\left(S^{2} \rtimes S^{1}\right) .
\end{aligned}
$$

First note that $|V|=1$ as explained in the proof of Theorem 8.1. This unique triangulation $T \in V$ has

$$
\operatorname{er}(T)=0.8056, \quad C_{27}(T)=87.01
$$

Let Triang $_{27}$ denote the finite, uniform probability space of all triangulations of closed 3-manifolds with 27 tetrahedra. Then Triang $_{27}$ has a partition

$$
\operatorname{Triang}_{27}=U \cup V \text {, }
$$

and the maximal mean commute time $C_{27}$ is a random variable on Triang $_{27}$. We have to determine the (empirical) conditional probabilities

$$
\mathbb{P}\left(U \mid C_{27} \leq 102\right), \quad \mathbb{P}\left(V \mid C_{27} \leq 102\right) .
$$

Since $|V|=1$ and this unique triangulation has $C_{27} \leq$ 102 , we have

$$
\mathbb{P}\left(C_{27} \leq 102 \mid V\right)=1
$$

According to the empirical density of $C_{27}$ on $S_{3}$,

$$
\mathbb{P}\left(C_{27} \leq 102 \mid U\right)=0.009
$$

By Bayes's theorem,

$$
\begin{aligned}
& \mathbb{P}\left(U \mid C_{27} \leq 102\right) \\
& \quad=\frac{\mathbb{P}\left(C_{27} \leq 102 \mid U\right) \mathbb{P}(U)}{\mathbb{P}\left(C_{27} \leq 102 \mid U\right) \mathbb{P}(U)+\mathbb{P}\left(C_{27} \leq 102 \mid V\right) \mathbb{P}(V)} \\
& \quad=\frac{0.009 \mathbb{P}\left(S^{3}\right)}{0.009 \mathbb{P}\left(S^{3}\right)+\mathbb{P}\left(S^{2} \rtimes S^{1}\right)} .
\end{aligned}
$$

Similarly for $\mathbb{P}\left(V \mid C_{27} \leq 102\right)$.

\subsection{Real Projective Space}

The empirical density of the random variable $C_{40}\left(S^{3}\right)$ (i.e., maximal mean commute time of random walks on triangulations of $S^{3}$ with 40 tetrahedra) is shown in Figure 5 , the density of $C_{40}\left(S^{2} \rtimes S^{1}\right)$ is shown in Figure 6,

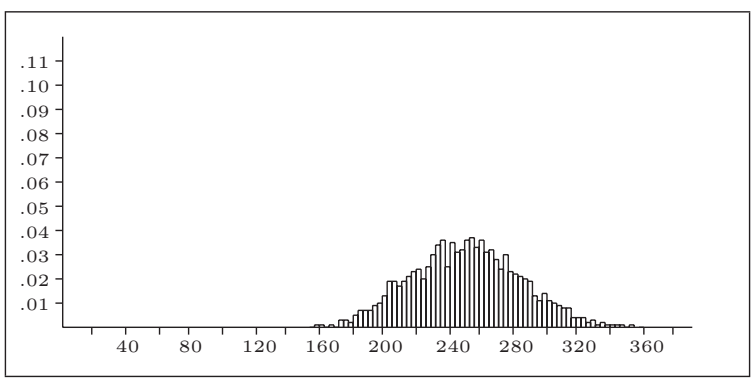

FIGURE 5. Commute time density on the 3-sphere with 40 tetrahedra.

while Figure 7 displays the density of $C_{40}\left(S^{2} \times S^{1}\right)$. The following table records the sample means:

\begin{tabular}{|c|r|}
\hline$M$ & $\bar{C}_{40}(M)$ \\
\hline$S^{3}$ & 250.1 \\
\hline$S^{2} \rtimes S^{1}$ & 180.9 \\
\hline$S^{2} \times S^{1}$ & 180 \\
\hline
\end{tabular}

The densities strongly distinguish $S^{3}$ from both $S^{2} \rtimes S^{1}$ and $S^{2} \times S^{1}$, but do not seem to detect a substantial difference between $S^{2} \rtimes S^{1}$ and $S^{2} \times S^{1}$. Heuristic 8.4 below relies on assuming the validity of the following conjecture in discrete geometry (see, for example, [Lutz 05]):

Conjecture 8.3. Walkup's vertex-minimal triangulation [Walkup 70] of $\mathbb{R} P^{3}$ is unique with $f$-vector $f=$ $(11,51,80,40)$.

Heuristic 8.4. Suppose an unknown manifold $M$ is triangulated with 40 tetrahedra. Let $C$ denote the maximal mean commute time associated with the combinatorial random walk on this triangulation. Then:

1. $C<120$ or $C>135$.

2. If $C>135$, then $M$ is $S^{3}, S^{2} \rtimes S^{1}$, or $S^{2} \times S^{1}$.

3. If $C<120$, then $M=\mathbb{R} P^{3}$.

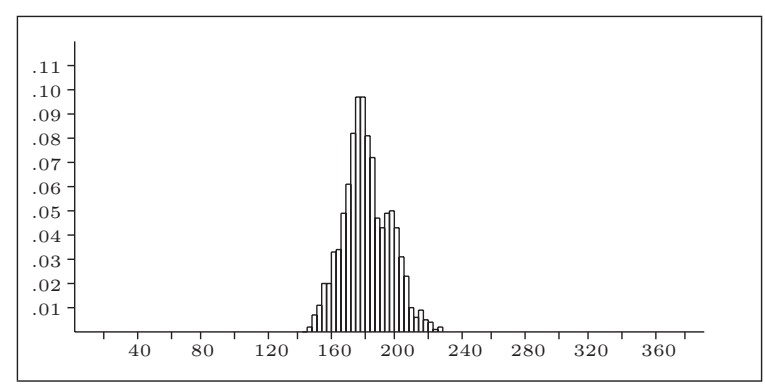

FIGURE 6. Commute time density on $S^{2} \rtimes S^{1}$ with 40 tetrahedra. 


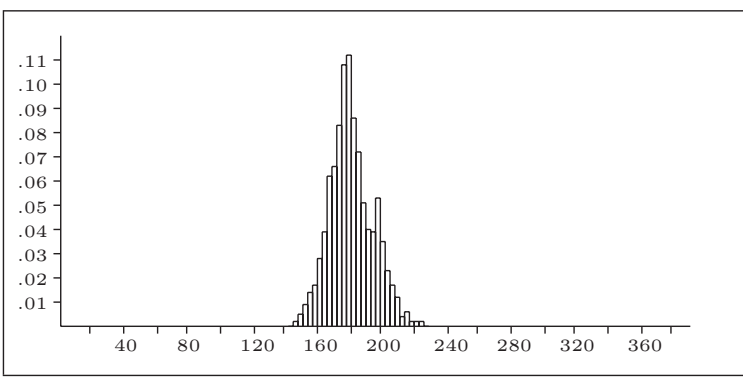

FIGURE 7. Commute time density on $S^{2} \times S^{1}$ with 40 tetrahedra.

4. Assume we know for instance that $C \leq 162$. Then $M$ is $S^{3}, S^{2} \rtimes S^{1}, S^{2} \times S^{1}$, or $\mathbb{R} P^{3}$ with probabilities

$$
\begin{aligned}
\mathbb{P}\left(S^{3} \mid C \leq 162\right) & =0.0023 \mathbb{P}\left(S^{3}\right) / p, \\
\mathbb{P}\left(S^{2} \rtimes S^{1} \mid C \leq 162\right) & =0.0957 \mathbb{P}\left(S^{2} \rtimes S^{1}\right) / p, \\
\mathbb{P}\left(S^{2} \times S^{1} \mid C \leq 162\right) & =0.0777 \mathbb{P}\left(S^{2} \times S^{1}\right) / p, \\
\mathbb{P}\left(\mathbb{R} P^{3} \mid C \leq 162\right) & =\mathbb{P}\left(\mathbb{R} P^{3}\right) / p,
\end{aligned}
$$

where

$$
\begin{aligned}
p= & 0.0023 \mathbb{P}\left(S^{3}\right)+0.0957 \mathbb{P}\left(S^{2} \rtimes S^{1}\right) \\
& +0.0777 \mathbb{P}\left(S^{2} \times S^{1}\right)+\mathbb{P}\left(\mathbb{R} P^{3}\right) .
\end{aligned}
$$

Verification of Heuristic 8.4: An argument similar to that given in the verification of Heuristic 8.2 shows that when $n=40, M$ has to be $S^{3}, S^{2} \rtimes S^{1}, S^{2} \times S^{1}$, or $\mathbb{R} P^{3}$. Here we have $40=f_{3}=f_{1}-f_{0} \geq 3 f_{0}+\gamma(M)$ with $\gamma(M) \geq 8$ if $M$ is not $S^{3}, S^{2} \rtimes S^{1}, S^{2} \times S^{1}$, or $\mathbb{R} P^{3}$. Thus $f_{0} \leq 10$, which contradicts the fact that any manifold not homeomorphic to $S^{3}, S^{2} \rtimes S^{1}$, or $S^{2} \times S^{1}$ needs at least 11 vertices to be triangulated.

Let

$$
\begin{aligned}
U & =\operatorname{Triang}_{40}\left(S^{3}\right), \\
V & =\operatorname{Triang}_{40}\left(S^{2} \rtimes S^{1}\right), \\
W & =\operatorname{Triang}_{40}\left(S^{2} \times S^{1}\right), \\
X & =\operatorname{Triang}_{40}\left(\mathbb{R} P^{3}\right) .
\end{aligned}
$$

Let Triang $_{40}$ denote the finite uniform probability space of all triangulations of closed 3-manifolds with 40 tetrahedra. Then Triang 40 has a partition

$$
\operatorname{Triang}_{40}=U \cup V \cup W \cup X,
$$

and the maximal mean commute time $C_{40}$ is a random variable on Triang 40 . We have to determine the (empirical) conditional probabilities

$$
\begin{aligned}
& \mathbb{P}\left(U \mid C_{40} \leq t\right), \quad \mathbb{P}\left(V \mid C_{40} \leq t\right), \\
& \mathbb{P}\left(W \mid C_{40} \leq t\right), \quad \mathbb{P}\left(X \mid C_{40} \leq t\right),
\end{aligned}
$$

for $t=135,162$.

Under the assumption that Conjecture 8.3 is correct, $X$ is a singleton: we have to show that if $T \in X$, then the $f$-vector of $T$ is $f=(11,51,80,40)$. The inequality

$$
f_{1} \geq 4 f_{0}+\gamma\left(\mathbb{R} P^{3}\right)=4 f_{0}+7,
$$

together with

$$
40=f_{3}=f_{1}-f_{0},
$$

implies that $f_{0} \leq 11$. On the other hand, Theorem 4.1 asserts that $f_{0} \geq 11$, so that $f_{0}=11$. Finally, general properties of 3 -manifold $f$-vectors (see $(4-1)$ ) yield $f_{1}=$ $51, f_{2}=80$.

Walkup's minimal triangulation $T$ of $\mathbb{R} P^{3}, X=\{T\}$, has maximal mean commute time $C_{40}(T)=119.42$. This establishes statement 2.

For $t=135$, according to the empirical densities for $S^{3}, S^{2} \rtimes S^{1}, S^{2} \times S^{1}$, shown in Figures $5-7$, we have

$$
\left\{T \in \operatorname{Triang}_{40}(M) \mid C_{40}(T)<135\right\}=\varnothing
$$

for $M=S^{3}, S^{2} \rtimes S^{1}, S^{2} \times S^{1}$. Statements 1 and 3 follow.

The probabilities that the commute time is below 162 are, according to the experimental data,

$$
\begin{aligned}
p_{U} & =\mathbb{P}\left(C_{40} \leq 162 \mid U\right)=0.0023, \\
p_{V} & =\mathbb{P}\left(C_{40} \leq 162 \mid V\right)=0.0957, \\
p_{W} & =\mathbb{P}\left(C_{40} \leq 162 \mid W\right)=0.0777, \\
p_{X} & =\mathbb{P}\left(C_{40} \leq 162 \mid X\right)=1 .
\end{aligned}
$$

Consequently, with

$$
p=p_{U} \mathbb{P}(U)+p_{V} \mathbb{P}(V)+p_{W} \mathbb{P}(W)+p_{X} \mathbb{P}(X),
$$

we obtain the following Bayes formulas: for the sphere,

$$
\mathbb{P}\left(U \mid C_{40} \leq 162\right)=p_{U} \mathbb{P}(U) / p
$$

the 3-dimensional Klein bottle,

$$
\mathbb{P}\left(V \mid C_{40} \leq 162\right)=p_{V} \mathbb{P}(V) / p
$$

for $S^{2} \times S^{1}$,

$$
\mathbb{P}\left(W \mid C_{40} \leq 162\right)=p_{W} \mathbb{P}(W) / p
$$

and for projective space,

$$
\mathbb{P}\left(X \mid C_{40} \leq 162\right)=p_{X} \mathbb{P}(X) / p
$$




\section{ACKNOWLEDGMENTS}

We are indebted to the monograph [Aldous and Fill 06] of D. Aldous and J. Fill on random walks on graphs, to F. Lutz's excellent survey article [Lutz 05], as well as to valuable remarks on a preliminary version of this article by two anonymous referees.

\section{REFERENCES}

[Aldous and Fill 06] D. Aldous and J. Fill. "Reversible Markov Chains and Random Walks on Graphs." Preprint available online (http://www.stat.berkeley.edu/ users/aldous/RWG/book.html).

[Altshuler and Steinberg 76] A. Altshuler and L. Steinberg. "An Enumeration of Combinatorial 3-Manifolds with Nine Vertices." Discrete Math. 16 (1976), 91-108.

[Bing 59] R. H. Bing. "An Alternative Proof That 3Manifolds Can Be Triangulated." Ann. Math. (2) 69 (1959), 37-65.

[Burton 04] B. A. Burton. "Face Pairing Graphs and 3Manifold Enumeration." J. Knot Theory and Its Ramifications 13:8 (2004), 1057-1101.

[Chandra et al. 89] A. Chandra et al. "The Electrical Resistance of a Graph Captures Its Commute and Cover Times." In Proc. 21st Annual ACM Symposium on Theory of Computing, pp. 574-586, 1989.

[Coppersmith et al. 96] D. Coppersmith, U. Feige, and J. Shearer. "Random Walks on Regular and Irregular Graphs." SIAM J. Discrete Math. 9 (1996), 301-308.

[Delaunay 28] B. Delaunay. "Sur la sphère vide." Proc. Internat. Congr. Math., Vol. 1, pp. 695-700, 1928.
[Leibon 99] G. Leibon. "Random Delaunay Triangulations, the Thurston-Andreev Theorem, and Metric Uniformization." PhD diss., University of California, San Diego, 1999 .

[Lickorish 99] W. B. R. Lickorish. "Simplicial Moves on Complexes and Manifolds." In Proceedings of the Kirbyfest, Geometry \& Topology Monographs 2, pp. 299-320, 1999.

[Lutz 05] F. H. Lutz. "Triangulated Manifolds with Few Vertices: Combinatorial Manifolds." arXiv math.CO/0506372, 2005.

[Moise 52] E. Moise. "Affine Structures in 3-Manifolds V: The Triangulation Theorem and Hauptvermutung." Ann. Math. (2) 56 (1952), 96-114.

[Munkres 84] J. R. Munkres. Elements of Algebraic Topology. Reading, MA: Addison-Wesley, 1984.

[Marsaglia and Zaman 91] G. Marsaglia and A. Zaman. "A New Class of Random Number Generators." Ann. Appl. Probab. 1:3 (1991), 462-480.

[Pachner 78] U. Pachner. "Bistellare Äquivalenz kombinatorischer Mannigfaltigkeiten." Arch. Math. 30 (1978), 8991.

[Pachner 87] U. Pachner. "Konstruktionsmethoden und das kombinatorische Homöomorphieproblem für Triangulationen kompakter semilinearer Mannigfaltigkeiten." Abh. Math. Sem. Univ. Hamburg 57 (1987), 69-86.

[Tutte 62] W. T. Tutte. "A Census of Planar Triangulations." Canad. J. Math. 14 (1962), 21-38.

[Walkup 70] D. W. Walkup. "The Lower Bound Conjecture for 3- and 4-Manifolds." Acta Math. 125 (1970), 75-107.

Markus Banagl, Mathematisches Institut, Universität Heidelberg, Im Neuenheimer Feld 288, 69120 Heidelberg, Germany (banagl@mathi.uni-heidelberg.de)

Received July 18, 2005; accepted in revised form January 17, 2006. 
